\title{
THE LMO-INVARIANT OF 3-MANIFOLDS OF RANK ONE AND THE ALEXANDER POLYNOMIAL
}

\author{
JENS LIEBERUM
}

\begin{abstract}
We prove that the LMO-invariant of a 3-manifold of rank one is determined by the Alexander polynomial of the manifold, and conversely, that the Alexander polynomial is determined by the LMO-invariant. Furthermore, we show that the Alexander polynomial of a null-homologous knot in a rational homology 3 -sphere can be obtained by composing the weight system of the Alexander polynomial with the Århus invariant of knots.
\end{abstract}

Mathematics Subject Classification (2000): 57M25, 57N65, 57M15.

Keywords: Alexander polynomial, finite type invariants, 3-manifolds, knots.

\section{INTRODUCTION}

In analogy with the theory of Vassiliev invariants of links, different notions of finite type invariants of 3-manifolds have been introduced. For integral homology spheres these different notions coincide with the original definition of Ohtsuki (Oht). The LMO-invariant $Z^{L M O}$ assembles all $\mathbb{Q}$-valued finite type invariants of integral homology spheres in a formal series and is therefore called a universal finite type invariant ([LMO, [Le1]). For connected closed manifolds $M$ the following is known about $Z^{L M O}$ :

\begin{tabular}{|l|l|}
\hline For $M$ with $\ldots$ & $Z^{L M O}$ is determined by and determines ... \\
\hline \hline rank $H_{1}(M)=0$ & all $\mathbb{Q}$-valued invariants of Goussarov and Habiro ([Habi]]) \\
\hline$H_{1}(M)=\mathbb{Z}$ & the Alexander polynomial (Theorem 1 of $[\mathrm{GaH}])$ \\
\hline $\operatorname{rank} H_{1}(M) \geq 2$ & the Casson-Walker-Lescop invariant $([\overline{\mathrm{HaB}}],[\mathrm{Hab}],[$ [Les] $)$ \\
\hline
\end{tabular}

In this article we fill in the missing puzzle piece for the interpretation of the LMOinvariant of manifolds of rank $\geq 1$ in terms of classical invariants. We prove the following generalization of Theorem 1 of [GaH].

Theorem 1. Let $M$ be a closed oriented 3-manifold of rank 1. Then the LMOinvariant $Z^{L M O}(M)$ is determined by the Alexander polynomial $\nabla(M)$, and conversely, $\nabla(M)$ is determined by $Z^{L M O}(M)$.

In the proof of Theorem 1 of $\mathrm{GaH}$ it was used that the Alexander polynomial $\nabla$ of links $L$ in $S^{3}$ can be obtained from the universal Vassiliev invariant $Z$ of links in $S^{3}$ via a map $W_{\nabla}$ as follows:

I would like to thank D. Bar-Natan, A. Beliakova, N. Habegger, T. Q. T. Le, and D. Thurston for helpful discussions. I thank the German Academic Exchange Service for financial support. Research at MSRI is supported in part by NSF grant DMS-9701755. 


$$
\frac{h}{e^{h / 2}-e^{-h / 2}} \nabla(L)_{\mid t^{1 / 2}=e^{h / 2}}=W_{\nabla} \circ Z(L) .
$$

We generalize Equation (11) by replacing $Z$ by the Århus invariant $\AA$ ( BGRT1) of knots in a rational homology sphere:

Theorem 2. Let $K$ be a null-homologous knot in a rational homology 3-sphere. Then

$$
\frac{h}{e^{h / 2}-e^{-h / 2}} \nabla(K)_{\mid t^{1 / 2}=e^{h / 2}}=W_{\nabla} \circ \AA(K) .
$$

Theorem 2 is an important ingredient in the proof of Theorem 1. Theorems 11 and 2 will be proven in Section 1 . In Sections 1 - 3 we prepare these proofs by recalling definitions and properties of the Alexander polynomial of links and manifolds, of unitrivalent diagrams and the map $W_{\nabla}$, and of the universal finite type invariants $Z, Z^{L M O}$ and $\AA$.

\section{The Alexander polynomial}

In this section we make preliminary definitions and recall some facts about the Alexander polynomial $\nabla$ from [Les].

All manifolds and submanifolds in this paper are oriented. Let $M$ be a rational homology 3 -sphere (meaning that $M$ is a connected closed manifold of dimension 3 with $H_{1}(M, \mathbb{Q})=0$ ). Let $K$ be a knot in $M$. Choose a tubular neighborhood $T$ of $K$. A meridian of $K$ is a simple closed curve $m$ on the boundary $\partial T$ of $T$ that is null-homologous in $T$. The curve $m$ is oriented by the right-hand rule. There exists a unique isomorphism $i_{K}: H_{1}(M \backslash K, \mathbb{Q}) \longrightarrow \mathbb{Q}$ that sends a meridian of $K$ to 1 . As a $\mathbb{Q}$-linear map $i_{K}$ is uniquely determined by the property that for any oriented surface $\Sigma \subset M$ with $\partial \Sigma \cap K=\emptyset$ the value $i_{K}(\partial \Sigma)$ is the intersection number of $K$ with $\Sigma$. For disjoint knots $K_{1}, K_{2}$ the linking number $\operatorname{lk}\left(K_{1}, K_{2}\right)$ is defined as $i_{K_{1}}\left(K_{2}\right)$. The linking number $\mathrm{lk}(.,$.$) is symmetric.$

Denote the number of components of a link $L$ by $|L|$. A framed link $L$ is a link with a simple closed curve $\mu_{i}$ on the boundary $\partial T_{i}$ of a tubular neighborhood $T_{i}$ of each component $K_{i}(i=1, \ldots,|L|)$. Inside of $T_{i}, \mu_{i}$ is homologous to $q_{i} K_{i}$ for some $q_{i} \in \mathbb{Z}$. The linking matrix $\left(l_{i j}\right)$ of $L$ is defined by $l_{i j}=\operatorname{lk}\left(K_{i}, \mu_{j}\right) / q_{j}$. The link $L$ has integral framing if all $q_{i}$ are 1 . The values $l_{i i} \in \mathbb{Q}$ are called framing of $K_{i}$. We denote by $M_{L}$ the manifold obtained by surgery on $L \subset M$.

Let $L \subset M$ be a null-homologous link. Then there exists an oriented connected surface $\Sigma$ embedded in $M$ such that $\partial \Sigma=L$. Any surface with this property is called a Seifert surface of $L$. Let $\Sigma^{ \pm}=\Sigma^{+} \cup \Sigma^{-}$be a tubular neighborhood of $\Sigma$ such that $\Sigma=\Sigma^{+} \cap \Sigma^{-}$and $\Sigma^{+}$lies on the positive side of $\Sigma$. The Seifert form of $\Sigma \subset M$ is the $\mathbb{Z}$-bilinear form $s: H_{1}(\Sigma) \times H_{1}(\Sigma) \longrightarrow \mathbb{Q}$ defined by sending homology classes $a$, $b$ to $\operatorname{lk}\left(A^{-}, B^{+}\right)$where $A^{-}$is a knot in $\Sigma^{-}$representing $a$ and $B^{+}$is a knot in $\Sigma^{+}$ representing $b$. In this section a matrix of $s$ with respect to an arbitrary basis of $H_{1}(\Sigma)$ is called a Seifert matrix of $\Sigma$ (later we will choose a particular basis of $H_{1}(\Sigma)$ ). Define the bilinear form $s^{*}$ by $s^{*}(a, b)=s(b, a)$. Then $s-s^{*}$ is the intersection form of $\Sigma$. Denote the transpose of a matrix $V$ by $V^{*}$. 
Proposition 3. Let $L$ be a null-homologous link in a rational homology sphere $M$. Choose a Seifert surface $\Sigma$ of $L$. Let $V$ be a Seifert matrix of $\Sigma$. Then

$$
\nabla(L)=\operatorname{det}\left(t^{1 / 2} V-t^{-1 / 2} V^{*}\right) \in\left(t^{1 / 2}-t^{-1 / 2}\right)^{|L|-1} \mathbb{Q}\left[\left(t^{1 / 2}-t^{-1 / 2}\right)^{2}\right] \subset \mathbb{Q}\left[t^{ \pm 1 / 2}\right]
$$

is an invariant of the pair $L \subset M$ up to homeomorphism; in particular it is an isotopy invariant of $L$.

Proposition 3 can be proven by using sign-determined Reidemeister torsion (see Proposition 2.3.13 of [Les], [Tur).

Up to sign the invariant $\nabla(L)$ can be described as follows. Let $N$ be a connected 3-manifold and let $\varphi: H_{1}(N) \longrightarrow \mathcal{Z}=\mathbb{Z}$ be a homomorphism. Let $\tilde{N}$ be the connected cover of $N$ corresponding to $\operatorname{Ker}(\varphi)$. Then $H_{1}(\tilde{N})$ is a module over the group ring $\mathbb{Z}[\mathcal{Z}] \cong \mathbb{Z}\left[t^{ \pm 1}\right]$. Let $J \subset \mathbb{Z}\left[t^{ \pm 1}\right]$ be the order ideal of $H_{1}(\widetilde{N})$. Let $\Delta_{\varphi}(N)$ be a generator of the smallest principal ideal containing $J$. Then $\Delta_{\varphi}(N)$ is unique up to multiplication by $\pm t^{i}$. For a link in a rational homology sphere $M$ we denote $\Delta_{\varphi}(M \backslash$ $L)$ by $\Delta(L)$, where $\varphi: H_{1}(M \backslash L) \longrightarrow \mathbb{Z}$ is given by the sum of the linking numbers with the components of $L$. The following lemma (see Proposition 2.3.13 of [Les]) relates $\nabla(L)$ and $\Delta(L)$.

Lemma 4. Let $L$ be a null-homologous link in a rational homology sphere $M$. Then there exists a unique $i \in \mathbb{Z}$ such that $t^{i / 2} \Delta(L)$ is invariant under the replacement of $t^{1 / 2}$ by $-t^{-1 / 2}$. For some $\epsilon \in\{ \pm 1\}$ we have $\epsilon t^{i / 2} \Delta(L)=\left|H_{1}(M)\right| \nabla(L)$.

Now consider a connected closed 3-manifold $N$ of rank 1 . Denote the quotient of $H_{1}(N)$ by its torsion subgroup $\operatorname{Tor}\left(H_{1}(N)\right)$ by $H_{1}^{\#}(N)$. Choose an isomorphism $\psi$ : $H_{1}^{\#}(N) \longrightarrow \mathbb{Z}$. Denote the composition of the canonical projection $H_{1}(N) \longrightarrow$ $H_{1}^{\#}(N)$ with $\psi$ by $\bar{\psi}$. The following two lemmas (see [Les], Section 5.1) allow to compare $\Delta_{\bar{\psi}}(N)$ with a knot invariant.

Lemma 5. Every connected closed 3-manifold $N$ of rank 1 can be obtained by 0framed surgery on a null-homologous knot $K$ in a rational homology sphere $M$. We then have $\operatorname{Tor}\left(H_{1}(N)\right) \cong H_{1}(M)$.

Lemma 6. Let $K$ be a null-homologous 0-framed knot in a rational homology 3sphere $M$. Then $\Delta(K)$ is equal to $\Delta_{\bar{\psi}}\left(M_{K}\right)$ up to multiplication by $\pm t^{i}$.

We see by Lemmas 4 , 5 and 6 that there exists $j \in \mathbb{Z}$ such that $t^{j / 2} \Delta_{\bar{\psi}}(N)$ is invariant under the replacement of $t^{1 / 2}$ by $-t^{-1 / 2}$. Furthermore, we can choose $\epsilon \in\{ \pm 1\}$ such that $\epsilon \Delta_{\bar{\psi}}(N)_{\mid t=1}=\left|H_{1}(\operatorname{Tor}(N))\right|>0$. Denote $\left(\epsilon t^{j / 2} /\left|H_{1}(\operatorname{Tor}(N))\right|\right) \Delta_{\bar{\psi}}(N)$ by $\nabla(N)$. The definition of $\nabla(N)$ does not depend on the choice of the isomorphism $\psi$ because $\nabla(N) \in \mathbb{Q}\left[\left(t^{1 / 2}-t^{-1 / 2}\right)^{2}\right]$. The invariant $\nabla$ satisfies

$$
\nabla(K)=\nabla\left(M_{K}\right)
$$

for all null-homologous 0 -framed knots $K$ in a rational homology sphere $M$. 


\section{UNITRIVALENT DiAGRAMS AND $W_{\nabla}$}

In this section we briefly recall facts about unitrivalent diagrams and use them to state properties of the Vassiliev invariants in the Alexander polynomial $\nabla$.

Let $\Gamma$ be a compact oriented 1-manifold whose boundary $\partial \Gamma$ is partitioned into two ordered sets called upper and lower boundary. Let $X$ be a set. A unitrivalent diagram with skeleton $\Gamma$ is a graph $D$ with distinguished subgraph $\Gamma$ such that all vertices of $D$ are either univalent or trivalent. Trivalent vertices not lying on $\Gamma$ are called internal and are oriented by a cyclic order of the incident edges. Univalent vertices are also called legs. Each leg of a unitrivalent diagram is labeled by an element of $X$. We allow connected components in $D$ that do not intersect $\Gamma$ whenever these components contain at least one trivalent vertex. Recall the definition of a $\mathbb{Q}$-vector space $\mathcal{A}(\Gamma, X)$ generated by unitrivalent diagrams modulo relations called $(S T U),(I H X)$, and $(A S)$ ([BN1]). When $\Gamma$ is equipped with additional information (for example: dots on circle-components of $\Gamma$, a set $Y$ in bijection with circle-components of $\Gamma$, a distinguished subset of the components of $\Gamma, \ldots)$, we require in the definition of $\mathcal{A}(\Gamma, X)$ that homeomorphisms between unitrivalent diagrams also preserve this additional data. The space $\mathcal{A}(\Gamma, X)$ is graded by half of the number of vertices of unitrivalent diagrams. Denote $\mathcal{A}(\Gamma, \emptyset)$ by $\mathcal{A}(\Gamma)$.

The invariants of $\ell$-component links in $S^{3}$ that are coefficients of $z^{i}=\left(t^{1 / 2}-t^{-1 / 2}\right)^{i}$ in $\nabla(L)$ induce linear forms $W_{i}: \mathcal{A}_{i}^{\ell} \longrightarrow \mathbb{Q}$ on the degree- $i$ part $\mathcal{A}_{i}^{\ell}$ of $\mathcal{A}^{\ell}:=\mathcal{A}\left(S^{1} \sqcup\right)$ (see Section 3 of $[\mathrm{BNG}]$ ). For $a$ in the completion of $\mathcal{A}^{\ell}$ by the degree, we define

$$
W_{\nabla}(a)=\sum_{i} W_{i}(a) h^{i} \in \mathbb{Q}[[h]] .
$$

It will follow from Theorem 2 and can also be seen directly that the Alexander polynomial of links in a rational homology sphere induces the same map $W_{\nabla}$ (see skein relation 2.3.16 of [Les, or Exercise 3.10 of $B \mathrm{BNG}$ ). The map $W_{\nabla}$ and its extensions to $\mathcal{A}(\Gamma, X)$ obtained from representations of the Lie superalgebra $\operatorname{gl}(1 \mid 1)$ have the following property (see Proposition 7.1 of [Vai], consider the element $\ominus$ of $\mathcal{A}(\emptyset)$ seperately).

Lemma 7. Let $D \in \mathcal{A}(\Gamma, X)$ be a unitrivalent diagram. Assume that $D$ has an internal vertex $u$ such that all edges incident to $u$ are connected to internal vertices. Then we have $W_{\nabla}(D)=0$.

Let $I_{x} \cong I:=[0,1](x \in X)$. Denote the disjoint union by $\sqcup$. For every partition of $\partial\left(\Gamma \sqcup \bigsqcup_{x \in X} I_{x}\right)$ into two ordered sets called upper and lower boundary there exists an isomorphism

$$
\chi_{X}: \mathcal{A}(\Gamma, X \sqcup Y) \longrightarrow \mathcal{A}\left(\Gamma \sqcup \bigsqcup_{x \in X} I_{x}, Y\right)
$$

given by the average over all permutations of putting $x$-labeled univalent vertices of a diagram on the corresponding skeleton component $I_{x} \cong I$ of $\Gamma \sqcup \bigsqcup_{x \in X} I_{x}$. The inverse of $\chi_{X}$ will be denoted by $\sigma_{X}$ and the set $X$ will not be specified when it is clear from the context. Obviously, there exists an isomorphism of $\mathcal{A}\left(\Gamma \sqcup \bigsqcup_{x \in X} I_{x}, Y\right)$ 
with a space $\mathcal{A}\left(\Gamma \sqcup \bigsqcup_{x \in X} S_{x}^{1^{*}}, Y\right)$, where the circles $S_{x}^{1^{*}}$ have a dot and are in bijection with the set $X$. Similarly, we have a surjective map from $\mathcal{A}\left(\bigsqcup_{x \in X} I_{x} \sqcup_{y \in Y} I_{y}, Z\right)$

to $\mathcal{A}\left(\bigsqcup_{x \in X} I_{x} \sqcup \bigsqcup_{y \in Y} S_{y}^{1}, Z\right)$ given by closing the intervals $I_{y}$ to form the circles $S_{y}^{1}$. Denote the composition of $\chi_{Y}$ with this surjective map by $\bar{\chi}_{Y}$.

An important special case is $\mathcal{A}\left(S^{1}\right) \cong \mathcal{A}\left(S^{1^{*}}, \emptyset\right) \cong \mathcal{A}(I)=: \mathcal{A}$ (see [BN1]). The space $\mathcal{A}$ is a commutative algebra with multiplication \# induced by the connected sum of the skeletons $S^{1}$ of diagrams (resp. by the concatenation of skeletons $I$ of diagrams). More generally, the connected sum of $S^{1}$ with any distinguished skeleton component $C$ of a unitrivalent diagram turns $\mathcal{A}(\Gamma \cup C, X)$ into an $\mathcal{A}$-module. Let $\overline{\mathcal{A}}$ be the quotient of $\mathcal{A}$ by the ideal generated by the element $\ominus$ and let $\pi: \mathcal{A} \longrightarrow \overline{\mathcal{A}}$ be the canonical projection. There exists a unique inclusion of algebras $i: \overline{\mathcal{A}} \longrightarrow \mathcal{A}$ with the property that $i(D)=D$ for all diagrams $D$ such that $D \backslash S^{1}$ is connected and $D$ contains an internal vertex ([BN1], Equation (5), Exercise 3.16). The map $P_{\text {defr }}=i \circ \pi: \mathcal{A} \longrightarrow \mathcal{A}$ is called deframing projection .

The disjoint union of unitrivalent diagrams turns $\mathcal{A}(\emptyset, X)$ into a commutative algebra and $\mathcal{A}(\Gamma, X)$ into an $\mathcal{A}(\emptyset, X)$-module. Important examples of diagrams in $\mathcal{A}(\emptyset, X)$ are so-called struts ${ }^{i} \frown^{j}$ with labels $i, j \in X$, and so-called wheels $\omega_{n}=\not \subset$ having $n$ internal vertices lying on a circle and $n$ univalent vertices with the same label ( $n=4$ in this example). Let $\mathcal{A}(\emptyset, X)_{\text {strut }} \subset \mathcal{A}(\emptyset, X)$ be the subalgebra generated by struts and $\mathcal{A}(\emptyset, X)_{\text {wh }}$ be the subalgebra generated by wheels. It is known that $\mathcal{A}(\emptyset, X)_{\text {strut }}$ is a polynomial algebra in the $n(n+1) / 2$ different struts $(n=|X|)$ and $\mathcal{A}(\emptyset, X)_{\text {wh }}$ is a polynomial algebra in wheels with an even number of univalent vertices. There exist unique projections from $\mathcal{A}(\Gamma, X)$ to $\mathcal{A}(\emptyset, X)_{\text {strut }}$ (resp. $\left.\mathcal{A}(\emptyset, X)_{\text {wh }}\right)$ that send all diagrams to 0 that have a connected component that is not a strut (resp. a wheel). Define $P_{\text {strut }}: \mathcal{A}\left(\Gamma \sqcup \bigsqcup_{x \in X} I_{x}, \emptyset\right) \longrightarrow \mathcal{A}(\Gamma, X)$ as the composition of $\sigma$ with the projection to $\mathcal{A}(\emptyset, X)_{\text {strut }} \subset \mathcal{A}(\Gamma, X)$. The map $P_{\text {strut }}$ descends to $\mathcal{A}\left(\Gamma \sqcup \bigsqcup_{x \in X} S_{x}^{1}, \emptyset\right)$ where the circle-components $S_{x}^{1}$ are in bijection with $X$. Define $P_{\text {wh }}: \mathcal{A} \longrightarrow \mathcal{A}(\emptyset,\{x\})$ as the composition of $\sigma \circ P_{\text {defr }}$ with the projection to $\mathcal{A}(\emptyset,\{x\})_{\text {wh }}$. We have $P_{\mathrm{wh}}(a \# b)=P_{\mathrm{wh}}(a) \sqcup P_{\mathrm{wh}}(b)$ for all $a, b \in \mathcal{A}$. The map $P_{\mathrm{wh}}$ is related to $W_{\nabla}$ as follows (see Vail, [Kril).

Lemma 8. For $D \in \mathcal{A}$ the value $W_{\nabla}(D)$ depends only on $P_{\mathrm{wh}}(D)$ and is determined by

$$
W_{\nabla}\left(D_{1} \# D_{2}\right)=W_{\nabla}\left(D_{1}\right) W_{\nabla}\left(D_{2}\right) \quad \text { and } \quad W_{\nabla}\left(\bar{\chi}\left(\omega_{2 n}\right)\right)=-2 h^{2 n} .
$$

Lemma 8 was used in proofs of the Melvin-Morton-Rozansky conjecture ([BNG]).

\section{UNIVERSAL FINITE TYPE INVARIANTS}

Recall from Section 3 of [LM2] that a non-associative framed tangle (or q-tangle) $T$ is a usual tangle with integral framing, except that $\operatorname{source}(T)$ and $\operatorname{target}(T)$ are equipped with parentheses on the sequences of \pm -symbols associated with the lower and upper boundary points of $T$. We denote by $Z$ the universal Vassiliev invariant of non-associative framed tangles (see [LM2]). Denote the underlying 1-manifold of a tangle $T$ (together with the partition of $\partial T$ into two ordered sets and possibly 
together with a decoration of $T$ such as dots, distinguished components, ...) by $\Gamma(T)$. Then the values $Z(T)$ lie in the completion of $\mathcal{A}(\Gamma(T))$ by the degree.

Let $\nu=Z(O)$ be the invariant of the trivial knot with 0 -framing. Let $T=L^{\prime} \cup T^{\prime \prime}$ be a diagram of a framed non-associative tangle where the components of the sublink $L^{\prime}$ of $T$ are in bijection with a set $X^{\prime}$ and each component of $L^{\prime}$ has a dot on its circle. Define $\check{Z}(T)$ as the connected sum of $Z(T)$ with $\nu^{\otimes\left|L^{\prime}\right|}$ along the components of $\Gamma\left(L^{\prime}\right)$. Cut the chord diagrams in $\check{Z}(T)$ at the dots and apply the isomorphism $\sigma_{X^{\prime}}$. The result lies in the completion of $\mathcal{A}\left(\Gamma\left(T^{\prime \prime}\right), X^{\prime}\right)$ and is called $\check{Z}^{\sigma}(T)$. The value $\check{Z}^{\sigma}(T)$ is not invariant under isotopies of the tangle represented by the diagram $T$. For tangles $T$ with dotted circles $L^{\prime}$ invariants $Z_{0}^{L M O}(T)$ and $\AA_{0}(T)$ of isotopy (that are also invariant under second Kirby moves along $L^{\prime}$ ) are obtained from $C=\check{Z}^{\sigma}(T)$ as follows (see [LMO, [Le2, BGRT2]).

Definition of $Z_{0}^{L M O}$ : The degree- $n$ part of $Z_{0}^{L M O}(T):=<C>$ is obtained from the degree $n+\left|L^{\prime}\right| n$ part of $C$ by forgetting the diagrams in $C$ that do not have exactly $2 n$ legs of each color $x \in X^{\prime}$, by summing over all the $((2 n-1) ! !)^{\left|L^{\prime}\right|}=\left((2 n) ! / 2^{n} n !\right)^{\left|L^{\prime}\right|}$ possible ways of gluing pairs of legs of diagrams in $C$ with the same label and by replacing circles that do not belong to $\Gamma\left(T^{\prime \prime}\right)$ by $-2 n$.

Definition of $\AA_{0}: \AA_{0}$ is only defined when the linking matrix $\left(l_{i j}\right)$ of $L^{\prime}$ is invertible (or equivalently, when $S_{L^{\prime}}^{3}$ is a rational homology sphere). Write $C$ in the form

$$
C=P \sqcup \exp \left(\frac{1}{2} \sum_{i, j \in X^{\prime}} l_{i j}{ }^{i} \frown^{j}\right)
$$

where $P$ contains no struts. Let $\left(l^{i j}\right)$ be the inverse matrix of $\left(l_{i j}\right)$. Then

$$
\AA_{0}(T):=<P, \exp \left(-\frac{1}{2} \sum_{i, j \in X^{\prime}} l^{i j}{ }_{\partial i} \smile_{\partial j}\right)>,
$$

where $\left\langle D_{1}, D_{2}>\right.$ is 0 if for some $i$ the number of $i$-labeled legs of $D_{1}$ is not equal to the number of $\partial i$-labeled legs of $D_{2}$, and is given by the sum of all ways of gluing all legs with $i$-labels to legs with $\partial i$-labels in the remaining case.

Let $L \subset M$ be a link in a 3-manifold. Represent $L \subset M$ by a diagram $L^{\prime} \cup L^{\prime \prime}$ of a link in $S^{3}$, such that $S_{L^{\prime}}^{3} \cong M$ and the image of $L^{\prime \prime}$ in $S_{L^{\prime}}^{3}$ is mapped to $L$ by this homeomorphism. Put a dot on each component of $L^{\prime}$. Two invariants $Z^{L M O}$ and $\AA$ of homeomorphisms of the pair $(M, L)$ are obtained from $Z_{0}^{L M O}\left(L^{\prime} \cup L^{\prime \prime}\right)$ and $\AA_{0}\left(L^{\prime} \cup L^{\prime \prime}\right)$ by normalization (making it invariant under the first Kirby move) as follows:

$$
\begin{aligned}
Z^{L M O}(L) & =Z_{0}^{L M O}\left(U_{+}\right)^{-\sigma_{+}} Z_{0}^{L M O}\left(U_{-}\right)^{-\sigma_{-}} Z_{0}^{L M O}\left(L^{\prime} \cup L^{\prime \prime}\right), \\
\AA(L) & =\AA_{0}\left(U_{+}\right)^{-\sigma_{+}} \AA_{0}\left(U_{-}\right)^{-\sigma_{-}} \AA_{0}\left(L^{\prime} \cup L^{\prime \prime}\right),
\end{aligned}
$$

where $U_{ \pm}$is the trivial knot with a dot and framing \pm 1 and $\sigma_{+}$(resp. $\sigma_{-}$) is the number of positive (resp. negative) eigenvalues of the linking matrix $\left(l_{i j}\right)$ of $L^{\prime}$. The invariants of the empty link $Z^{L M O}(\emptyset)$ and $\AA(\emptyset)$ are also denoted by $Z^{L M O}(M)$ and $\AA(M)$, respectively. The series $\AA_{0}\left(U_{ \pm}\right)$have degree-0 term 1 . Therefore Lemma $\square$ implies 


$$
W_{\nabla} \circ \AA_{0}\left(L^{\prime} \cup L^{\prime \prime}\right)=W_{\nabla} \circ \AA(L) .
$$

We will make use of the following result of [BGRT3] (Equation (7) follows from Proposition 1.2 of [BGRT3] in the same way as Theorem 1 of [BGRT3]):

$$
\AA(L)=\left|H_{1}(M)\right|^{-\operatorname{deg}} Z^{L M O}(L),
$$

where $\left|H_{1}(M)\right|^{- \text {deg }}$ denotes the operation of multiplying diagrams of degree $m$ by $\left|H_{1}(M)\right|^{-m}$.

Let us recall some notation used in Lemma 9 below. Let $T$ be a non-associative framed tangle $T$ with a distinguished subset $T$ of its components. Denote by $d(T)$ the non-associative framed tangle given by replacing each component in $\tilde{T}$ by two copies that are parallel with respect to the framing. The symbols $a \in\{+,-\}$ in source $(T)$ (resp. target $(T))$ that belong to $\tilde{T}$ are replace by $(a$ a $)$ in source $(d(T))$ (resp. target $(d(T)))$. Define $s(T)$ by reversing the orientation of each component in $\tilde{T}$. Define $\epsilon(T)$ by deleting $\tilde{T}$. Now let $D$ be a unitrivalent diagram $D$ with a distinguished subset $\tilde{\Gamma}$ of its skeleton components. Define $d(D)$ by replacing each skeleton component in $\tilde{\Gamma}$ by two copies, and by summing over all ways of lifting vertices of $D$ that lie on $\tilde{\Gamma}$ to the new skeleton. Define $s(D)$ by reversing the orientation of the components in $\tilde{\Gamma}$ and by multiplying with $\prod_{C \in \tilde{\Gamma}}(-1)^{n_{C}}$ where $n_{C}$ is the number of vertices lying on the skeleton component $C$ of $D$. If $n_{C}>0$ for some component $C$ of $\tilde{\Gamma}$, then define $\epsilon(D)=0$. Define $\epsilon(D)$ by deleting the components in $\tilde{\Gamma}$ in the remaining case. The composition $T_{1} \circ T_{2}$ of non-associative tangles $T_{1}, T_{2}$ with source $\left(T_{1}\right)=\operatorname{target}\left(T_{2}\right)$ is defined by placing $T_{1}$ on the top of $T_{2}$. For diagrams $D_{i}$ in $\mathcal{A}\left(\Gamma\left(T_{i}\right)\right)$ a composition $D_{1} \circ D_{2}$ is defined similarly. In the following lemma we state generalizations of well-known properties of $Z$.

Lemma 9. Let $T, T_{1}, T_{2}$ be non-associative tangles with dotted circles.

(1) Assume that some of the components of $T$ without dots are distinguished. Then we havet

$$
d\left(\AA_{0}(T)\right)=\AA_{0}(d(T)) \quad, \quad s\left(\AA_{0}(T)\right)=\AA_{0}(s(T)) \quad, \quad \epsilon\left(\AA_{0}(T)\right)=\AA_{0}(\epsilon(T)) .
$$

(2) Assume that $\operatorname{source}\left(T_{1}\right)=\operatorname{target}\left(T_{2}\right)$. Then

(3) We have

$$
\AA_{0}\left(T_{1} \circ T_{2}\right)=\AA_{0}\left(T_{1}\right) \circ \AA_{0}\left(T_{2}\right) .
$$

$$
\AA_{0}(T)=\bar{\chi}_{Y}((\exp (P))
$$

where $P$ is a series of connected diagrams in $\mathcal{A}(\emptyset, Y)$ and $Y$ is a set in bijection with the components of $T$ without dots.

\footnotetext{
${ }^{1} \mathrm{As}$ in [LM3 we must assume for the first property of $\AA_{0}$ that an even associator is used in the definition of $Z$. This causes no restrictions in Theorems 11 and 2 because for links $L$, the invariants $\AA(L)$ and $Z^{L M O}(L)$ do not depend on the choice of an associator.
} 
The proof of Lemma 9 is straightforward. Statements similar to Lemma 9 hold for $\AA(L)$.

\section{Proofs of Theorems 1 and 2}

Recall from Equation (11) that for links $L$ in $S^{3}$ we have $c \nabla(L)_{\mid t^{1 / 2}=e^{h / 2}}=W_{\nabla} \circ Z(L)$ with $c=h /\left(e^{h / 2}-e^{-h / 2}\right)$. Equation (11) is proven in [LM1 and [BNG by showing that $W_{\nabla} \circ Z$ satisfies a skein relation and $W_{\nabla} \circ Z(O)=c$. With the methods of this proof one can show directly that $W_{\nabla} \circ \AA$ satisfies the same skein relation for links in a rational homology sphere and $W_{\nabla} \circ \AA(O)=c$, but this does not imply Theorem 2 . In this section we present a proof of Theorem 2 based on Equation (11). Then we prove Theorem 1 by using Theorem 2 .

Let $L=L^{\prime} \cup L^{\prime \prime}$ be a framed link in a rational homology sphere $M$. Denote the components of $L^{\prime}$ (resp. $L^{\prime \prime}$ ) by $K_{x}$ with $x \in X^{\prime}$ (resp. $x \in X^{\prime \prime}$ ) and their framings by $\mu_{x}$. For $x, y \in X^{\prime} \cup X^{\prime \prime}$ let $l_{x y}=\operatorname{lk}\left(\mu_{x}, K_{y}\right)$ be linking numbers in $M$, let the submatrix corresponding to $L^{\prime}$ be invertible and denote its inverse by $\left(l^{x y}\right)_{x, y \in X^{\prime}}$. In the following lemma we recall how the linking numbers transform under surgery.

Lemma 10. For $i, j \in X^{\prime \prime}$ the linking numbers $\tilde{l}_{i j}=\operatorname{lk}\left(\mu_{i}, K_{j}\right)$ of $L^{\prime \prime} \subset M_{L^{\prime}}$ are given by

$$
\tilde{l}_{i j}=l_{i j}-\sum_{x, y \in X^{\prime}} l_{i x} l^{x y} l_{y j} .
$$

Proof. Denote the meridians of the components of $L$ by $m_{x}$. In $H_{1}\left(M \backslash\left(L^{\prime} \cup L^{\prime \prime}\right), \mathbb{Q}\right)$ the framings $\mu_{y}$ can uniquely be expressed as $\mu_{y}=\sum_{j \in X^{\prime} \cup X^{\prime \prime}} l_{y j} m_{j}$. This implies for $x \in X^{\prime}$ that

$$
\sum_{y \in X^{\prime}} l^{x y} \mu_{y}=m_{x}+\sum_{y \in X^{\prime}, j \in X^{\prime \prime}} l^{x y} l_{y j} m_{j} .
$$

In $H_{1}\left(M_{L^{\prime}} \backslash L^{\prime \prime}, \mathbb{Q}\right)=H_{1}\left(M \backslash\left(L^{\prime} \cup L^{\prime \prime}\right), \mathbb{Q}\right) /\left(\mu_{x}\right)_{x \in X^{\prime}}$ we obtain the following unique expression of $\mu_{i}\left(i \in X^{\prime \prime}\right)$ in terms of the meridians $m_{j}\left(j \in X^{\prime \prime}\right)$ of $L^{\prime \prime} \subset M_{L^{\prime}}$ :

$$
\mu_{i}=\sum_{j \in X^{\prime} \cup X^{\prime \prime}} l_{i j} m_{j}=\sum_{j \in X^{\prime \prime}} l_{i j} m_{j}-\sum_{x \in X^{\prime}, y \in X^{\prime}, j \in X^{\prime \prime}} l_{i x}{ }^{x y} l_{y j} m_{j} .
$$

This implies the lemma.

The following lemma tells us that the linking numbers of a link $L \subset M$ can be recovered from $P_{\text {strut }}(\AA(L))$.

Lemma 11. Let $L$ be a link with integral framing in a rational homology sphere $M$. Let the components of $L$ be in bijection with a set $X$. Let $\left(\tilde{l}_{i j}\right)_{i, j \in X}$ be the linking matrix of L. Then

$$
P_{\text {strut }}(\AA(L))=\exp \left(\frac{1}{2} \sum_{i, j \in X} \tilde{l}_{i j}{ }^{i} \frown^{j}\right) .
$$


Proof. Choose a diagram of $L^{\prime} \cup L^{\prime \prime} \subset S^{3}$ such that $\left(S_{L^{\prime}}^{3}, L^{\prime \prime}\right) \cong(M, L)$ and put dots on the components of $L^{\prime}$. Let $\left(l_{x y}\right)_{x, y \in X^{\prime} \cup X^{\prime \prime}}$ be the linking matrix of $L^{\prime} \cup L^{\prime \prime}$ and let $\left(l^{x y}\right)_{x, y \in X^{\prime}}$ be the inverse of the linking matrix of $L^{\prime}$. Then for a series $P$ (resp. $\tilde{P}$ ) of diagrams in $\mathcal{A}\left(\emptyset, X^{\prime} \cup X^{\prime \prime}\right)$ (resp. in $\mathcal{A}\left(\emptyset, X^{\prime \prime}\right)$ ) that contains no struts and has degree-0-term 1 , we have

$$
\begin{gathered}
\check{Z}^{\sigma}\left(L^{\prime} \cup L^{\prime \prime}\right)=\bar{\chi}_{X^{\prime \prime}}\left(P \sqcup \exp \left(\frac{1}{2} \sum_{x, y \in X^{\prime} \cup X^{\prime \prime}} l_{x y}{ }^{x} \frown^{y}\right)\right) \text { and } \\
\AA_{0}\left(L^{\prime} \cup L^{\prime \prime}\right)=\bar{\chi}_{X^{\prime \prime}}\left(<P \sqcup \exp \left(\frac{1}{2} \sum_{i, j \in X^{\prime \prime}} l_{i j}{ }^{i} \frown^{j}+\sum_{i \in X^{\prime \prime}, x \in X^{\prime}} l_{i x}{ }^{i} \frown^{x}\right),\right. \\
\left.\quad \exp \left(-\frac{1}{2} \sum_{x, y \in X^{\prime}} l^{x y}{ }_{\partial x} \smile_{\partial y}\right)>\right) \\
=\bar{\chi}_{X^{\prime \prime}}\left(\tilde{P} \sqcup \exp \left(\frac{1}{2} \sum_{i, j \in X^{\prime \prime}} l_{i j}{ }^{i} \frown^{j}-\frac{1}{2} \sum_{i, j \in X^{\prime \prime}, x, y \in X^{\prime}} l_{i x} l^{x y} l_{y j}{ }^{i} \frown^{j}\right)\right) .
\end{gathered}
$$

Since $P_{\text {strut }}(\AA(L))=P_{\text {strut }}\left(\AA_{0}\left(L^{\prime} \cup L^{\prime \prime}\right)\right)$, Lemma 11 follows from Lemma 10 .

For technical reasons we fix a representative of each homeomorphism-class of connected compact surfaces with boundary. We call this representative $\Sigma$ a standard surface and equip it with a decomposition into a single vertex $v \cong I \times I$ (also called coupon) with bands $B_{i} \cong I \times I$ that are glued along $I \times\{0,1\}$ to the upper boundary $I \times\{1\}$ of $v$. Call the part $I \times\{0\}$ of $v$ its distinguished lower boundary. We orient the cores $I \times\{1 / 2\}$ of the bands $B_{i}\left(i=1, \ldots\right.$, rank $\left.H_{1}(\Sigma)\right)$. An example is shown on the left side of Figure 1 .
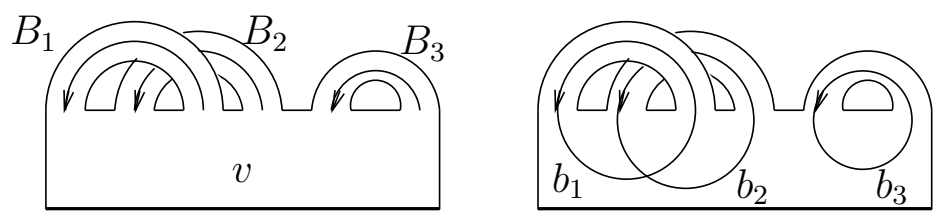

Figure 1. A standard surface $\Sigma$ and a basis $\left(b_{i}\right)$ of $H_{1}(\Sigma)$

We associate a basis of $H_{1}(\Sigma)$ to the ribbon graph decomposition of $\Sigma$ as shown in Figure 1 by an example. The orientation of $b_{i}$ is determined by the orientation of the core of the band $B_{i}$. An embedding of a standard surface into $R^{2} \times I$ is an example of a ribbon graph in the sense of Section 8 of [KaT]. Ribbon graphs without vertices can canonically be identified with framed tangles. We will use this identification in the following.

From now on we use the term Seifert matrix of a Seifert surface $\Sigma \subset M$ always with respect to a basis of $H_{1}(\Sigma)$ obtained by identifying $\Sigma$ with a standard surface in some freely chosen way. We use the same basis for a matrix of the intersection form of $\Sigma$. 
Keylemma 12. Let $K$ be a knot in a rational homology sphere $M$ bounding a Seifert surface $\Sigma$. Let $V$ be a Seifert matrix of $\Sigma$. Then the power series $W_{\nabla} \circ \AA(K)$ depends only on $V$. The coefficient of $h^{i}$ in this series is a polynomial in the entries of $V$.

Let us prepare the proof of Keylemma 12. We will make some statements more generally for links instead of knots. Let $V=\left(v_{i j}\right)$ be a Seifert matrix. Choose a null-homologous link $L$ in a rational homology sphere $M$ with Seifert surface $\Sigma$ and Seifert matrix $V$. The homeomorphism type of $\Sigma$ is determined by the similarity type of $V-V^{*}$. There exists a link with integral framing $\tilde{L} \subset M$ such that $M_{\tilde{L}}=S^{3}$. The link $\tilde{L}$ can be chosen to be disjoint from $\Sigma$ because changing crossings between $L=\partial \Sigma$ and $\tilde{L}$ preserves the property that $M_{\tilde{L}}=S^{3}$. Therefore $\Sigma \subset M$ can be obtained from a surface $\Sigma^{\prime \prime} \subset S^{3}$ by surgery along a link $L^{\prime} \subset S^{3} \backslash \Sigma^{\prime \prime}$. The identification of $\Sigma$ with a standard surface induces an identification of $\Sigma^{\prime \prime}$ with a standard surface. In a diagram of $L^{\prime} \cup \Sigma^{\prime \prime}$ the vertex $v$ of $\Sigma^{\prime \prime}$ can be pulled downwards, such that the diagram $L^{\prime} \cup \Sigma^{\prime \prime}$ is of the form $\left(L^{\prime} \cup T_{1}^{\prime \prime}\right) \circ T_{2}^{\prime \prime}$ where $T_{2}^{\prime \prime}$ is a planar diagram of a neighborhood of $v$ and the distinguished lower boundary of $v$ is the lowest part of the diagram. Put dots on the components of $L^{\prime}$. An example is shown in Figure 2 .

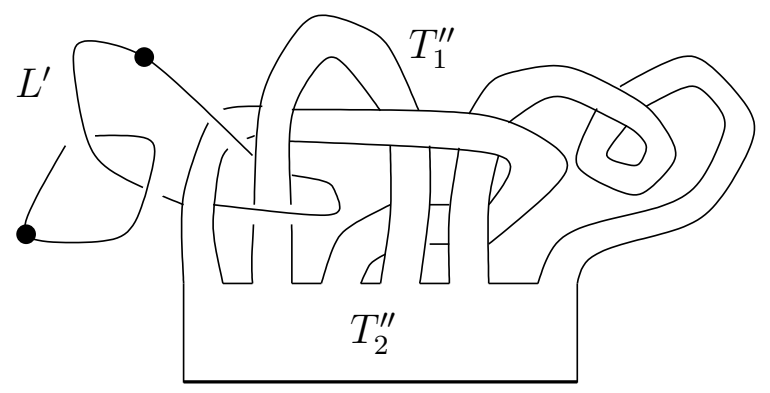

Figure 2. A diagram of $L^{\prime} \cup \Sigma^{\prime \prime}$

The components of $T_{1}^{\prime \prime}$ are in bijection with the set $X^{\prime \prime}=\left\{1, \ldots\right.$, rank $\left.H_{1}(\Sigma)\right\}$. Regard $T_{1}^{\prime \prime}$ as a non-associative framed tangle with parentheses of the form $((((\ldots))$.$) .)$ on source $\left(T_{1}^{\prime \prime}\right)$. Let $F=\left(f_{i j}\right)=V-V^{*}$ be the matrix of the intersection form of $\Sigma$ and let $U=\left(u_{i j}\right)=1 / 2\left(V+V^{*}\right)=V-1 / 2 F$.

Lemma 13. With the notation from above we have

$$
P_{\text {strut }}\left(\AA_{0}\left(L^{\prime} \cup T_{1}^{\prime \prime}\right)\right)=\exp \left(\frac{1}{2} \sum_{i, j \in X^{\prime \prime}} u_{i j}{ }^{i} \frown^{j}\right) .
$$

Proof. Let $K_{i}^{+}$(resp. $K_{i}^{-}$) be a knot in the upper part $\Sigma^{+} \subset M$ (resp. in the lower part $\left.\Sigma^{-} \subset M\right)$ of a tubular neighborhood of $\Sigma$ representing the $i$-th basis element of $H_{1}(\Sigma)$. Let the knot $K_{i}^{-} \cong K_{i}^{+}$have the framing $\operatorname{lk}\left(K_{i}^{-}, K_{i}^{+}\right)=v_{i i}$ induced by the surface $\Sigma$. First consider $i \neq j \in X^{\prime \prime}$. Define $P_{i j}$ as the composition of $P_{\text {strut }}$ with the projection to the part containing only powers of the strut ${ }^{i} \frown^{j}$. Lemma 11 implies that $P_{i j}\left(\AA\left(K_{i}^{-} \cup K_{j}^{+}\right)\right)=\exp \left(v_{i j}{ }^{i}{ }^{j}\right)$. Represent $K_{i}^{-} \cup K_{j}^{+} \subset M$ by a surgery diagram $\left(L^{\prime} \cup S_{1}^{\prime \prime}\right) \circ S_{2}^{\prime \prime}$ where the tangle $S_{1}^{\prime \prime}$ consists of the $i$-th and $j$-th framed strands of $T_{1}^{\prime \prime}$ and $S_{2}^{\prime \prime}$ is a 0 -framed tangle consisting of two intervals close to $T_{2}^{\prime \prime}$. See Figure 3 
for an example (compare Figures 11 and 2). In this figure the dotted line separates $S_{2}^{\prime \prime}$ from $L^{\prime} \cup S_{1}^{\prime \prime}$ and is not a part of the diagram.

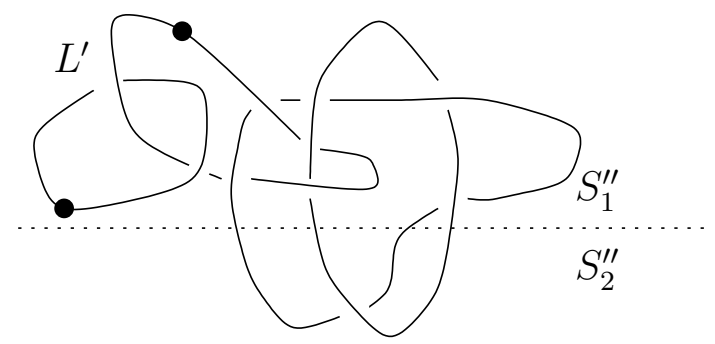

Figure 3. A surgery diagram $\left(L^{\prime} \cup S_{1}^{\prime \prime}\right) \circ S_{2}^{\prime \prime}$ of $K_{1}^{-} \cup K_{2}^{+}$

We have $\AA_{0}\left(S_{2}^{\prime \prime}\right)=Z\left(S_{2}^{\prime \prime}\right)$ and the explicit description of $Z$ (see [LM2) implies

$$
P_{\text {strut }}\left(\AA_{0}\left(S_{2}^{\prime \prime}\right)\right)=\exp \left((1 / 2) f_{i j}{ }^{i} \frown^{j}\right) .
$$

Observe the following property of $P_{i j}$ :

$$
P_{i j}\left(\AA_{0}\left(L^{\prime} \cup S_{1}^{\prime \prime}\right) \circ \AA_{0}\left(S_{2}^{\prime \prime}\right)\right)=P_{i j}\left(\AA_{0}\left(L^{\prime} \cup S_{1}^{\prime \prime}\right)\right) \sqcup P_{i j}\left(\AA_{0}\left(S_{2}^{\prime \prime}\right)\right) .
$$

The last two formulas and Part (2) of Lemma 9 imply

$$
P_{i j}\left(\AA_{0}\left(L^{\prime} \cup S_{1}^{\prime \prime}\right)\right)=\exp \left(\left(v_{i j}-f_{i j} / 2\right)^{i} \frown^{j}\right)=\exp \left(u_{i j}{ }^{i}{ }^{j}\right) .
$$

Using Lemma 9 for $\epsilon$ we see that $P_{i j}\left(\AA\left(L^{\prime} \cup T_{1}^{\prime \prime}\right)\right)=\exp \left(u_{i j}{ }^{i} \frown^{j}\right)$. For $i=j$ Lemma 11 implies $P_{i i}\left(\AA\left(K_{i}^{ \pm}\right)\right)=\exp \left((1 / 2) v_{i i}{ }^{i} \frown^{i}\right)=\exp \left((1 / 2) u_{i i}{ }^{i} \frown^{i}\right)$. We apply Lemma 9 for $\epsilon$ as above and obtain $P_{i i}\left(\AA_{0}\left(L^{\prime} \cup T_{1}^{\prime \prime}\right)\right)=\exp \left((1 / 2) u_{i i}{ }^{i} \frown^{i}\right)$. By Part (3) of Lemma 9 we have $P_{\text {strut }}\left(\AA_{0}\left(L^{\prime} \cup T_{1}^{\prime \prime}\right)\right)=\bigsqcup_{i \leq j} P_{i j}\left(\AA_{0}\left(L^{\prime} \cup T_{1}^{\prime \prime}\right)\right)$ which completes the proof.

Starting from $V$ we made a lot of choices in the definition of $T_{1}^{\prime \prime}$. Since $\AA(L)$ is an invariant only the choice of $L \subset M$ can influence $W_{\nabla} \circ \AA(L)$. Now we are ready to show that for knots $L$ the invariant $W_{\nabla} \circ \AA(L)$ depends only on $V$.

Proof of Keylemma 19. We use the notation from above. For suitable distinguished components of $T_{1}^{\prime \prime}$ and of $d\left(T_{1}^{\prime \prime}\right)$ the tangle $s\left(d\left(T_{1}^{\prime \prime}\right)\right)$ coincides with the part of the framed oriented boundary of $\Sigma^{\prime \prime}$ that belongs to $T_{1}^{\prime \prime}$. Let $T_{3}^{\prime \prime}$ be the part of the framed oriented boundary of $\Sigma^{\prime \prime}$ that belongs to $T_{2}^{\prime \prime}$. We regard $T_{3}^{\prime \prime}$ as a non-associative tangle with $\operatorname{target}\left(T_{3}^{\prime \prime}\right)=\operatorname{source}\left(s\left(d\left(T_{1}^{\prime \prime}\right)\right)\right)$. The invariant $Z\left(T_{3}^{\prime \prime}\right)=\AA_{0}\left(T_{3}^{\prime \prime}\right)$ depends only on rank $H_{1}(\Sigma)$. Since we know that the Seifert matrix $V$ is chosen with respect to a basis induced by a standard surface, the definition of the map $\chi_{X^{\prime \prime}}: \mathcal{A}\left(\emptyset, X^{\prime \prime}\right) \longrightarrow$ $\mathcal{A}\left(\Gamma\left(T_{1}^{\prime \prime}\right), \emptyset\right)$ depends only on $V$ (see Equation (3)). We will show below that for knots $L$ all terms in $\AA_{0}\left(L^{\prime} \cup T_{1}^{\prime \prime}\right)$ that contain an internal vertex do not contribute to $W_{\nabla}(\AA(L))$. Equation (6), Lemma 9 and Lemma 13 then imply 


$$
\begin{aligned}
W_{\nabla}(\AA(L)) & =W_{\nabla}\left(\AA_{0}\left(L^{\prime} \cup \partial \Sigma^{\prime \prime}\right)\right) \\
& =W_{\nabla}\left(s\left(d\left(\AA_{0}\left(L^{\prime} \cup T_{1}^{\prime \prime}\right)\right)\right) \circ Z\left(T_{3}^{\prime \prime}\right)\right) \\
& =W_{\nabla}\left(s\left(d\left(\chi_{X^{\prime \prime}}\left(P_{\text {strut }}\left(\AA_{0}\left(L^{\prime} \cup T_{1}^{\prime \prime}\right)\right)\right)\right)\right) \circ Z\left(T_{3}^{\prime \prime}\right)\right) \\
& =W_{\nabla}\left(s \circ d \circ \chi_{X^{\prime \prime}}\left(\exp \left(\frac{1}{4} \sum_{i, j \in X^{\prime \prime}}\left(v_{i j}+v_{j i}\right)^{i} \frown^{j}\right)\right) \circ Z\left(T_{3}^{\prime \prime}\right)\right) .
\end{aligned}
$$

This will show that $W_{\nabla}(\AA(L))$ is determined by the Seifert matrix $V$. Obviously, the coefficients of $h^{i}$ in $W_{\nabla}(\AA(L))$ are polynomials of degree $\leq i$ in the entries of $V$. This will prove the keylemma.

It remains to consider diagrams $D$ in $\AA_{0}\left(L^{\prime} \cup T_{1}^{\prime \prime}\right)$ with an internal vertex $u$. In $s(d(D))$ each of the edges incident to $u$ is either connected to another internal vertex or appears twice, namely as the difference of the two ways of lifting it to the skeleton $\Gamma\left(s\left(d\left(T_{1}^{\prime \prime}\right)\right)\right)$. We represent this difference by a box in Figure 4 .

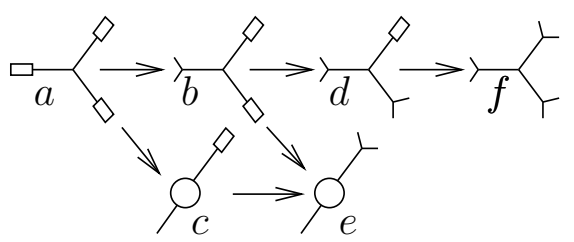

FiguRE 4. Replacing differences of univalent vertices by internal vertices

A neighborhood of the internal vertex $u$ looks like in one of the possibilities (a)-(f) in Figure 4 . When we push a lifted vertex in the box along the circle $\Gamma\left(\partial \Sigma^{\prime \prime}\right)$, then it will finally cancel with the second lifted vertex. By the (STU)-relation we can replace a box in Figure 4 by a sum of diagrams with an additional internal vertex. More precisely, a part of the diagram looking like in (a), (b), (c), or (d) in Figure 4 is replaced by a sum of diagrams where a neighborhood of $u$ looks like in diagrams that can be reached by following a directed arrow in Figure 4 . When we apply this procedure to all boxes, we will finally end up with possibilities (e) and (f). By Lemma 7 all diagrams that have a subdiagram as in (e) or (f) are sent to 0 by $W_{\nabla}$.

Let us recall a fact about knots (and links) in $S^{3}$ (see Proposition 8.7 of [BuZ]).

Fact 14. Let $V$ be a $n \times n$-matrix over $\mathbb{Z}$ such that $V-V^{*}$ is a matrix of the intersection form of a surface. Then $V$ is a Seifert matrix of a link in $S^{3}$.

Since for all Seifert forms $s$ the intersection form of $\Sigma$ is equal to $s-s^{*}$, we see that Seifert forms of a fixed surface $\Sigma$ are a subset of an affine space whose associated $\mathbb{Q}$ vector space are symmetric $\mathbb{Z}$-bilinear forms on $H_{1}(\Sigma)$ with values in $\mathbb{Q}$. By Fact 14 Seifert forms of Seifert surfaces $\Sigma$ in $S^{3}$ are a lattice of full rank in this affine space.

Proof of Theorem 6. By Proposition 3 and Keylemma 12 the coefficients of $h^{i}$ in the two power series $\frac{h}{e^{h / 2}-e^{-h / 2}} \nabla(K)_{\mid t^{1 / 2}=e^{h / 2}}$ and $W_{\nabla} \circ \AA(K)$ only depend on a Seifert 
matrix $V$ of a knot $K$ and are polynomials $p_{i}$ and $q_{i}$ in the entries of $V$. By Equation (1) we have $p_{i}(V)=q_{i}(V)$ for all Seifert matrices of knots in $S^{3}$. Fact 14 implies that $p_{i}=q_{i}$ for all $i$.

The following lemma is a straightforward extension of a result of [GaH].

Lemma 15. Let $K$ be a 0 -framed knot in a rational homology sphere $M$. Then any of the series $Z^{L M O}\left(M_{K}\right), W_{\nabla} \circ Z^{L M O}(K), W_{\nabla} \circ \AA(K)$ can be computed from any other of these series.

Sketch of proof. The invariants $Z^{L M O}$ and $\AA$ of $K \subset M$ differ only by normalization (see Equation (7)). Let $C=Z^{L M O}(K)$. Then we have $Z^{L M O}\left(M_{K}\right)=\langle\sigma(\nu \# C)\rangle$ with $\nu=Z(O)$. The following four steps show that $W_{\nabla}(C)$ can be calculated from $Z^{L M O}\left(M_{K}\right)$ and vice versa. This will complete the proof.

1) $W_{\nabla}(C)$ depends only on the wheel-part $P_{\mathrm{wh}}(C)$ of $C$ (Lemma 8 ).

2) $P_{\mathrm{wh}}(C)$ can be calculated from $W_{\nabla}(C)$ because $P_{\mathrm{wh}}(C)=\exp (P)$ where $P$ is a formal series of connected wheels (see Part (3) of Lemma 9), $W_{\nabla}(C)=\exp \left(W_{\nabla}(P)\right)$, and $W_{\nabla}$ is injective on connected wheels (Lemma 8).

3) $\sigma(\nu \# C)$ contains no struts because $K$ is 0 -framed (see Lemma 11 and Equation (7)). All remaining non-vanishing diagrams in $\mathcal{A}(\emptyset,\{x\})$ have at least as many internal vertices as univalent vertices. This implies that $Z^{L M O}\left(M_{K}\right)$ depends only on $P_{\mathrm{wh}}(\nu \# C)=P_{\mathrm{wh}}(\nu) \sqcup P_{\mathrm{wh}}(C)$.

4) The map $\langle\cdot\rangle$ is injective on wheels (see [GaH], Lemma 3.1, use the $\mathrm{sl}_{2}$-weight system on $\mathcal{A}(\emptyset)$ to see that $\langle\cdot>$ is injective on connected wheels). Therefore $P_{\mathrm{wh}}(\nu) \sqcup P_{\mathrm{wh}}(C)$ can be calculated from $Z^{L M O}\left(M_{K}\right)=<P_{\mathrm{wh}}(\nu) \sqcup P_{\mathrm{wh}}(C)>$. $P_{\mathrm{wh}}(\nu)$ is invertible.

Now we prove the main result of this paper.

Proof of Theorem 11. By Lemmas 5 and 15 and by Equation (2) it is sufficient to show that for a null-homotopic knot $K$ in a rational homology sphere each of the invariants $\nabla(K)$ and $W_{\nabla} \circ \AA(K)$ can be computed from the other one. This statement follows from Theorem 2 .

\section{REFERENCES}

[Ale] J. W. Alexander, Topological invariants of knots and links, Trans. Amer. Math. Soc. 30 (1928), 275-306.

[BGRT1] D. Bar-Natan, S. Garoufalidis, L. Rozansky and D. P. Thurston, The Arhus integral of rational homology 3-spheres I: A highly non trivial flat connection on $S^{3}$, Selecta Math., to appear.

[BGRT2] D. Bar-Natan, S. Garoufalidis, L. Rozansky and D. P. Thurston, The Arhus integral of rational homology 3-spheres II: Invariance and Universality, Selecta Math., to appear.

[BGRT3] D. Bar-Natan, S. Garoufalidis, L. Rozansky and D. P. Thurston, The Arhus integral of rational homology 3-spheres III: The Relation with the Le-Murakami-Ohtsuki Invariant, preprint, August 1998, see math/9808013.

[BN1] D. Bar-Natan, On the Vassiliev knot invariants, Topology 34 (1995), 423-472.

[BNG] D. Bar-Natan and S. Garoufalidis, On the Melvin-Morton-Rozansky conjecture, Invent. Math. 125 (1996), 103-133.

[BuZ] G. Burde and H. Zieschang, Knots, Walter de Gruyter, 1985. 
[Con] J. H. Conway, An enumeration of knots and links, and some of their algebraic properties, Computational Problems in Abstract Algebra, Pergamon (1970), 329-358.

[GaH] S. Garoufalidis and N. Habegger, The Alexander polynomial and finite type 3-manifold invariants, Math. Annalen, to appear.

[Hab] N. Habegger, A computation of the universal quantum 3-manifold invariant for manifolds of rank greater than 2, Université de Nantes preprint, Dec. 1996.

[HaB] N. Habegger and A. Beliakova, The Casson-Walker-Lescop invariant as a quantum 3manifold invariant, Université de Nantes and University of Bern preprint, Aug. 1997, see q-alg/9708029.

[Habi] K. Habiro, Claspers and finite type invariants of links, Geometry and Topology 4, No. 1 (2000), 1-83.

[KaT] C. Kassel and V. Turaev, Chord diagram invariants of tangles and graphs, Duke Math. J. 92 (1998), 497-552.

[Kri] A. Kricker, Alexander-Conway limits of many Vassiliev weight systems, J. of Knot Th. and its Ramif. 6(5) (1997), 687-714.

[Les] C. Lescop, Global surgery formula for the Casson-Walker invariant, Annals of Math. Studies No. 140, Princeton Univ. Press 1996.

[Le1] T. Q. T. Le, An invariant of integral homology 3-spheres which is univeral for all finite type invariants, Solitons, geometry and topology: on the crossroad, (V. Buchstaber and S. Novikov, eds.) AMS Translations Series 2, Providence.

[Le2] T. Q. T. Le, On denominators of the Kontsevich integral and the universal perturbative invariant of 3-manifolds, University of Buffalo preprint, April 1997.

[LM1] T. Q. T. Le and J. Murakami, Kontsevich's integral for the Homfly polynomial and relations between values of multiple zeta functions, Topology and its Appl. 62 (1995), 193-206.

[LM2] T. Q. T. Le and J. Murakami, The universal Vassiliev-Kontsevich invariant for framed oriented links, Comp. Math. 102 (1996), 41-64.

[LM3] T. Q. T. Le and J. Murakami, Parallel version of the universal Vassiliev-Kontsevich invariant, J. Pure and appl. Algebra 121 (1997), 271-291.

[LMO] T. Q. T. Le, J. Murakami and T. Ohtsuki, On a universal quantum invariant of 3-manifolds, Topology 37-3 (1998), 539-574.

[Oht] T. Ohtsuki, Finite type invariants of integral homology 3-spheres, J. of Knot Th. and its Ramif. 5(1) (1996), 101-115.

[Tur] V. G. Turaev, Reidemeister torsion in knot theory, Russ. Math. Surv. 41, No. 1 (1986), 119-182.

[Vai] A. Vaintrob, Melvin-Morton conjecture and primitive Feynman diagrams, University of Utah preprint, May 1996, see q-alg/9605028.

Jens Lieberum, Mathematical Sciences Research Institute, 1000 Centennial Drive, BERKELEY, CA 94720 - 5070, USA

E-mail address: lieberum@msri.org 